\title{
On structural properties of Helbing's gas-kinetic traffic flow model*
}

\author{
I. Necoara, B. De Schutter, and H. Hellendoorn
}

If you want to cite this report, please use the following reference instead:

I. Necoara, B. De Schutter, and H. Hellendoorn, "On structural properties of Helbing's gas-kinetic traffic flow model," Proceedings of the 2004 American Control Conference, Boston, Massachusetts, pp. 5508-5513, June-July 2004.

Delft Center for Systems and Control

Delft University of Technology

Mekelweg 2, 2628 CD Delft

The Netherlands

phone: +31-15-278.24.73 (secretary)

URL: https: //www.dcsc.tudelft.nl 


\title{
On Structural Properties of Helbing's Gas-Kinetic Traffic Flow Model
}

\author{
Ion Necoara, Bart De Schutter, Hans Hellendoorn
}

\begin{abstract}
There exist several types of models that describe the evolution of traffic flow on freeways and urban roads. In this paper we focus on some structural properties of one particular traffic flow model: the macroscopic, second-order gas-kinetic traffic flow model of Helbing. We will show that the model has two families of characteristics for the shock wave solutions: one characteristic is slower and the other one is faster than the average vehicle speed. Corresponding to the slower characteristic there are 1-shocks and 1-rarefaction waves, corresponding to the faster characteristic there are 2shocks and 2-rarefaction waves. We also derive the formulas for the solution of the Riemann problem associated with this model in the equilibrium case, proving that the solution of this problem with density and flow non-negative in the initial condition on either side of the discontinuity cannot give rise to negative flow or density later on.
\end{abstract}

\section{INTRODUCTION}

Traffic flow models play an important role in both today's traffic research and in many traffic applications such as traffic flow prediction, incident detection, and traffic control. Each traffic application requires specific features of the traffic model. As one of the goals of our research is to apply model-based traffic control, we are particularly interested in macroscopic traffic flow models since these models are best suited for implementation in traffic control systems. Macroscopic models offer an excellent trade-off between accuracy on the one hand and simulation speed on the other hand.

One of the most well-known traffic flow models is the Lighthill-Whitham-Richards (LWR) model [1], which is a macroscopic first-order model. Payne [2] came up with a second-order traffic model. Later on Papageorgiou et al. introduced an improved version of this model [3]. In this paper we discuss yet another macroscopic second-order model, which is based on gas-kinetic equations with a nonlocal term as proposed by Helbing [4].

We analyze the structural properties of the shock and rarefaction wave solutions of Helbing's model, because understanding these properties, helps to improve this area of research: more insight into the structural properties of the Helbing's model allows us to select appropriate and efficient numerical methods for traffic simulation using this model. Subsequently, this can then be used in model-based predictive traffic control approaches.

This paper is organized as follows. To make the paper self-contained, a brief review of the Helbing's model is

I. Necoara, B. De Schutter, J. Hellendoorn are with Delft Center for Systems and Control, Delft University of Technology, Mekelweg 2, 2628 CD Delft, The Netherlands \{i.necoara, b.deschutter, j.hellendoorn\}@dcsc.tudelft.nl presented in Section II. The new contributions of this paper start with Section III, where we discuss the structural properties of the shock wave solution. In Section IV we present the structural properties of the rarefaction waves solution. We show that the Helbing's model has two families of characteristics for the shock wave solutions: one characteristic is slower, and the other one is faster than the average vehicle speed. Corresponding to the slower characteristic there are 1-shocks and 1-rarefaction waves, the behavior of which is similar to that of shocks and rarefaction waves in the LWR model. Corresponding to the faster characteristic there are 2-shocks and 2-rarefaction waves, which behave differently from the previous one, in the sense that the information, in principle, travels faster than average vehicle speed. Finally, in Section V we discuss the solution of the Riemann problem associated with the Helbing's model, proving that the solution of this problem with density and flow non-negative in the initial condition on either side of the discontinuity cannot give rise to negative flow or density later on.

\section{HELBING'S TRAFFIC FLOW MODEL}

Because the number of vehicles is conserved, all macroscopic traffic flow models are based on the continuity equation, which expresses the relation between the rates of change of the density $\rho(x, t)$ with respect to time $t$ and of the flow $Q(x, t)$ with respect to space $x$ :

$$
\frac{\partial}{\partial t} \rho(x, t)+\frac{\partial}{\partial x} Q(x, t)=0
$$

To describe time-varying and spatially varying average velocities $V(x, t)$ such as those that occurs in traffic jams or stop-and-go traffic we need a dynamic velocity equation. Gas-kinetic equations for the average velocity have been proposed in a number of previous publications such as [5], [6], [7]. Because we are interested in macroscopic quantities we can integrate those equations to derive formulas for the first moment. For all these models the velocity equation can be written as ${ }^{1}$

$$
\frac{\partial V}{\partial t}+V \frac{\partial V}{\partial x}+\frac{1}{\rho} \frac{\partial P}{\partial x}=\frac{V_{\mathrm{eq}}-V}{\tau},
$$

where $P$ is the traffic pressure, defined as $P(x, t)=$ $\rho(x, y) \theta(x, t)$, with $\theta$ the velocity variance (see also (2) below), and where $V_{\mathrm{eq}}$ is the dynamical equilibrium velocity toward which the average velocity of vehicles relaxes.

\footnotetext{
${ }^{1}$ For the sake of brevity and simplicity of notation we will from now on omit the arguments $x$ and $t$ in the formulation of the partial differential equations.
} 
Helbing proposes the following Euler-like equation with a non-local term for the average vehicle velocity:

$$
\begin{aligned}
\frac{\partial V}{\partial t}+ & \underbrace{V \frac{\partial V}{\partial x}}_{\text {transport }}+\underbrace{\frac{1}{\rho} \frac{\partial P}{\partial x}}_{\text {pressure }}= \\
& \underbrace{\frac{V_{0}-V}{\tau}}_{\text {acceleration }}-\underbrace{\frac{V_{0}\left(\theta+\theta_{\mathrm{a}}\right)}{\tau A\left(\rho_{\max }\right)}\left(\frac{\rho_{\mathrm{a}} T}{1-\rho_{\mathrm{a}} / \rho_{\max }}\right)^{2} B\left(\delta_{v}\right)}_{\text {braking }} .
\end{aligned}
$$

The braking term is a non-local term that models braking in response to the traffic situation downstream at the interaction point $x_{\mathrm{a}}=x+\gamma\left(1 / \rho_{\max }+T V\right)$ with $1<\gamma<2$ a model parameter, $\rho_{\max }$ is the maximum density, and $T$ is the average time headway. In equation (1) we also have a Boltzmann factor of the form

$$
B\left(\delta_{v}, S\right)=\delta_{v} \frac{e^{-z^{2} / 2}}{\sqrt{2 \pi}}+\left(1+\delta_{v}^{2}\right) \int_{-\infty}^{\delta_{v}} \frac{e^{-z^{2} / 2}}{\sqrt{2 \pi}} d z,
$$

with $\delta_{v}=\frac{V-V_{\mathrm{a}}}{\sqrt{\theta-\theta_{\mathrm{a}}}}$, which takes into account the velocity and variance at the actual position $x$ and the interaction point $x_{\mathrm{a}}$ respectively. Based on empirical data, Helbing observed that the velocity variance $\theta$ is a density-dependent fraction $A(\rho)$ of the squared velocity:

$$
\theta(x, t)=A(\rho(x, t)) V^{2}(x, t),
$$

where $A(\rho)$ is the Fermi function

$$
A(\rho)=A_{0}+\Delta A\left(1+\tanh \left(\frac{\rho-\rho_{\mathrm{c}}}{\Delta \rho}\right)\right),
$$

where $A_{0}$ and $A_{0}+2 \Delta A$ are about the variance factors for free and congested traffic, $\rho_{\mathrm{c}}$ is of the order of the critical density for the transition from free to congested traffic, and $\Delta \rho$ is the width of the transition.

To summarize, the equations of Helbing's model are:

$$
\begin{aligned}
& \frac{\partial \rho}{\partial t}+\frac{\partial Q}{\partial x}=0, \\
& \frac{\partial V}{\partial t}+V \frac{\partial V}{\partial x}+\frac{1}{\rho} \frac{\partial P}{\partial x}=\frac{V_{\mathrm{eq}}-V}{\tau},
\end{aligned}
$$

where the equilibrium velocity is written as

$$
V_{\mathrm{eq}}=V_{0}\left(1-\frac{\theta+\theta_{a}}{A\left(\rho_{\max }\right)}\left(\frac{\rho_{\mathrm{a}} T}{1-\rho_{\mathrm{a}} / \rho_{\max }}\right)^{2} B\left(\delta_{v}\right)\right) .
$$

\section{HUGONIOT LOCUS AND SHOCKS}

A desirable property of the traffic model equations (4)(6) is that they can be formulated in terms of a system of conservation equations (i.e., a time-dependent system of nonlinear partial differential equations with a particular simple structure) but with a source term:

$$
\frac{\partial u}{\partial t}+\frac{\partial f(u)}{\partial x}=S(u)
$$

with state variables $u=\left[\begin{array}{ll}\rho & Q\end{array}\right]^{T}$, and with flux function $f$ and source term $S$ given by:

$$
f(u)=\left[\begin{array}{c}
Q \\
\frac{Q^{2}}{\rho}(1+A(\rho))
\end{array}\right], S(u)=\left[\begin{array}{c}
0 \\
\frac{\rho V_{\mathrm{eq}}-Q}{\tau}
\end{array}\right],
$$

where we used the relation $Q=\rho V$ between flow $Q$, density $\rho$, and average velocity $V$.

The pressure has the form: $P=\rho A(\rho) V^{2}=\frac{Q^{2}}{\rho} A(\rho)$, which implies that in matrix representation using the Jacobian

$J \stackrel{\text { def }}{=} \frac{\partial f}{\partial u}=\left[\begin{array}{cc}0 & 1 \\ -\frac{Q^{2}}{\rho^{2}}\left(1+A(\rho)-\rho \frac{d}{d \rho} A(\rho)\right) & 2 \frac{Q}{\rho}(1+A(\rho))\end{array}\right]$

we have

$$
\frac{\partial}{\partial t}\left[\begin{array}{l}
\rho \\
Q
\end{array}\right]+J(u) \cdot \frac{\partial}{\partial x}\left[\begin{array}{l}
\rho \\
Q
\end{array}\right]=\left[\begin{array}{c}
0 \\
\frac{\rho V_{\mathrm{eq}}-Q}{\tau}
\end{array}\right] .
$$

When we compute the eigenvalues of the Jacobian, and using again the relation $V=\frac{Q}{\rho}$, we get

$$
\lambda_{1,2}(u)=V\left(1+A(\rho) \pm \sqrt{A^{2}(\rho)+A(\rho)+\rho \frac{d}{d \rho} A(\rho)}\right) .
$$

Note that in our case, the system (8) is strictly hyperbolic (i.e., for any value of $u$ the eigenvalues of the Jacobian $J$ are real and distinct).

Corresponding to the two distinct eigenvalues given by (9) we have two linearly independent eigenvectors

$$
r_{1,2}(u)=\left[\begin{array}{c}
1 \\
\lambda_{1,2}(u)
\end{array}\right] \text {. }
$$

Assumption A1: As Helbing recommends in [4] for qualitative considerations, $A(\rho)$ can be chosen to be constant. We adopt this assumption henceforth because it simplifies our computations. We choose for $A(\rho)$ the value $c \stackrel{\text { def }}{=} A_{0}+\Delta A \approx$ 0.028 (which is the value around critical density where we have large oscillations of the speed).

With Assumption A1 the formulas for pressure $P$, flux $f$, and eigenvalues $\lambda_{p}(p=1,2)$ are

$$
\begin{aligned}
& P=c \rho V^{2}=c \frac{Q^{2}}{\rho}, \quad f(u)=\left[\begin{array}{c}
Q \\
\frac{Q^{2}}{\rho}(1+c)
\end{array}\right], \\
& \lambda_{1,2}(u)=V\left(1+c \pm \sqrt{c^{2}+c}\right)=c_{1,2} V,
\end{aligned}
$$

where we denote $c_{1} \stackrel{\text { def }}{=} 1+c-\sqrt{c^{2}+c} \in(0,1)$ and $c_{2} \stackrel{\text { def }}{=}$ $1+c+\sqrt{c^{2}+c}>1$. Note that $\lambda_{1}<\lambda_{2}$. Now let us study different kinds of shocks arising from the system and determine and characterize the conditions under which a pair of states $u_{\text {left }}=\left[\begin{array}{ll}\rho_{\text {left }} & Q_{\text {left }}\end{array}\right]^{T}, u_{\text {right }}=\left[\begin{array}{ll}\rho_{\text {right }} & Q_{\text {right }}\end{array}\right]^{T}$ can be connected by a shock ${ }^{2}$.

\footnotetext{
${ }^{2}$ The reason for selecting the subscripts "left" and "right" will become clear in Section V.
} 
First, note that in short time intervals the shocks arising from (7) are the same as those arising from

$$
\frac{\partial}{\partial t}\left[\begin{array}{l}
\rho \\
Q
\end{array}\right]+\left[\begin{array}{cc}
0 & 1 \\
-\frac{Q^{2}}{\rho^{2}}(1+c) & 2 \frac{Q}{\rho}+(1+c)
\end{array}\right] \cdot \frac{\partial}{\partial x}\left[\begin{array}{l}
\rho \\
Q
\end{array}\right]=0
$$

i.e., the source term becomes zero (this can be done when traffic operations are in equilibrium but also because the relaxation term $\frac{\rho V_{\mathrm{eq}}-Q}{\tau}$ is finite, so that its effect in short time intervals can be neglected compared to the effect caused by the infinite space derivatives of $\rho$ and $Q$ at the shock). Because we have two characteristics (eigenvalues), two kinds of shocks arise from (10): we call them 1-shock and 2-shock respectively.

Let us fix a state $u_{\text {left }}$, and determine the set of states $u_{\text {right }}$ that can be connected by a discontinuity (called Hugoniot locus) to the point $u_{\text {left }}$. For this, the Rankine-Hugoniot jump condition [8] must hold:

$$
f\left(u_{\text {right }}\right)-f\left(u_{\text {left }}\right)=s \cdot\left(u_{\text {right }}-u_{\text {left }}\right),
$$

where $s$ is the propagation speed of the discontinuity along the road. This speed is known in traffic flow engineering as congestion velocity and expresses the fact that the propagation of a shock depends on both flow and density in the neighboring region of a shock. Filling out the expression for $f$, yields the following solutions in terms of $\tilde{\rho}$

$$
Q_{\text {right } 1,2}=Q_{\text {left }} \frac{1 \pm\left(\rho_{\text {right }}-\rho_{\text {left }}\right) \sqrt{\frac{c^{2}+c}{\rho_{\text {right }} \rho_{\text {left }}}}}{1-\frac{\rho_{\text {right }}-\rho_{\text {left }}}{\rho_{\text {right }}}(1+c)},
$$

and the corresponding shock speed

$$
S_{\text {right } 1,2}=Q_{\text {left }} \frac{\frac{1+c}{\rho_{\text {right }}} \pm \sqrt{\frac{c^{2}+c}{\rho_{\text {right }} \rho_{\text {left }}}}}{1-\frac{\rho_{\text {right }}-\rho_{\text {left }}}{\rho_{\text {right }}}(1+c)},
$$

where the \pm signs give two solutions, one for each family of characteristic fields.

Now, we have to choose in formula (11) the sign for the 1-shock and for the 2-shock respectively. After some computations (see [9] for more details), we find that for the 1 -shock we must choose the minus sign and for the 2-shock the plus sign.

Remark: We can see that each of the characteristic fields is genuinely nonlinear, which means that

$\nabla \lambda_{p}(u) \cdot r_{p}(u)=c_{p}\left(c_{p}-1\right) \frac{Q}{\rho^{2}} \neq 0 \quad$ for all $u=\left[\begin{array}{l}\rho \\ Q\end{array}\right] \neq 0$,

where $\nabla \lambda_{p}=\left[\begin{array}{ll}\frac{\partial \lambda_{p}}{\partial \rho} & \frac{\partial \lambda_{p}}{\partial Q}\end{array}\right]$

In defining the Hugoniot locus above, we have ignored the question of whether a given discontinuity is physically relevant. Lax [10] proposed an entropy condition to systems of equations that are genuinely nonlinear: the jump in the $p$ th field (from state $u_{\text {left }}$ to $u_{\text {right }}$ ) is admissible only if

$$
\lambda_{p}\left(u_{\text {left }}\right)>s>\lambda_{p}\left(u_{\text {right }}\right),
$$

where $s$ is the shock speed. Now suppose we connect $u_{\text {left }}$ to $u_{\text {right }}$ by a 1 -shock, then we get the following relations:

$$
c_{1} \frac{Q_{\text {left }}}{\rho_{\text {left }}}>s>c_{1} \frac{Q_{\text {right }}}{\rho_{\text {right }}} .
$$

We obtain after few steps (see [9] for details)

$$
Q_{\text {left }}<Q_{\text {right }} \text {. }
$$

So for the 1-shock we have obtained the following: $Q_{\text {left }}<$ $Q_{\text {right }}$ and we should take the minus sign in formulas (11) and (12). Combining these two conditions we can show that we must have $\rho_{\text {right }}>\rho_{\text {left }}$ (see also [9]).

In summary, a 1-shock satisfies:

$$
\mathrm{S} 1:\left\{\begin{array}{c}
Q_{\text {right }}=Q_{\text {left }} \frac{1-\left(\rho_{\text {right }}-\rho_{\text {left }}\right) \sqrt{\frac{c^{2}+c}{\rho_{\text {right }} \rho_{\text {left }}}}}{1-\frac{\rho_{\text {right }}-\rho_{\text {left }}}{\rho_{\text {right }}}(1+c)}, \\
\rho_{\text {right }}>\rho_{\text {left }} .
\end{array}\right.
$$

Now let us see what is the interpretation of a 1-shock. Do the drivers on the overage really behave as described by $\mathrm{S} 1$ ? If we consider the fundamental diagram that relates speed and density (see [11]), then we see that the condition $\rho_{\text {right }}>\rho_{\text {left }}$ implies $V_{\text {right }}<V_{\text {left }}$ i.e., the drivers that enters that shock reduce their speed abruptly which coincides with real traffic behavior.

Similarly, one can show that for a 2-shock the following inequality holds: $Q_{\text {left }}>Q_{\text {right }}$, and we should take the plus sign in (11) and (12) as we saw before, which implies that $\rho_{\text {right }}<\rho_{\text {left }}$ So for a 2 -shock we have

$$
\mathrm{S} 2:\left\{\begin{array}{c}
Q_{\text {right }}=Q_{\text {left }} \frac{1+\left(\rho_{\text {right }}-\rho_{\text {left }}\right) \sqrt{\frac{c^{2}+c}{\rho_{\text {right }} \rho_{\text {left }}}}}{1-\frac{\rho_{\text {right }}-\rho_{\text {left }}}{\rho_{\text {right }}}(1+c)}, \\
\rho_{\text {right }}<\rho_{\text {left }} .
\end{array}\right.
$$

Now we can sketch the Hugoniot locus in the phase plane, retaining only the points $\tilde{u}$ that can be connected to $\hat{u}$ by an entropy-satisfying shock, discarding the entropy-violating shocks (see Figure 1). Any right state $u_{\text {right }}=\left[\rho_{\text {right }} Q_{\text {right }}\right]^{T}$ can be connected to a left state $u_{\text {left }}=\left[\rho_{\text {left }} Q_{\text {left }}\right]^{T}$ by a $1-$ shock if the right state falls on the $\mathrm{S} 1$ curve that passes through $\left[\rho_{\text {left }} Q_{\text {left }}\right]^{T}$ and similarly by a 2 -shock if the right state falls on the $\mathrm{S} 2$ curve that passes through $\left[\rho_{\text {left }} Q_{\text {left }}\right]^{T}$. We can see from Figure 1 that the Hugoniot locus terminates at the origin and there are no states with $u_{\text {right }}<0$ that can be connected to $u_{\text {left }}$ by a propagating discontinuity; therefore, the model does not produce negative density and flow at the point of discontinuity (as others traffic flow models that do so, see [12]).

\section{RAREFACTION WAVES}

For the LWR model it is known that when the left characteristic is slower than the right characteristic a fan of rarefaction waves results. This property is also valid in the Helbing's model. If the two characteristic fields satisfy

$$
\lambda_{p}\left(u_{\text {left }}\right)<\lambda_{p}\left(u_{\text {right }}\right) \quad \text { for } p=1,2,
$$




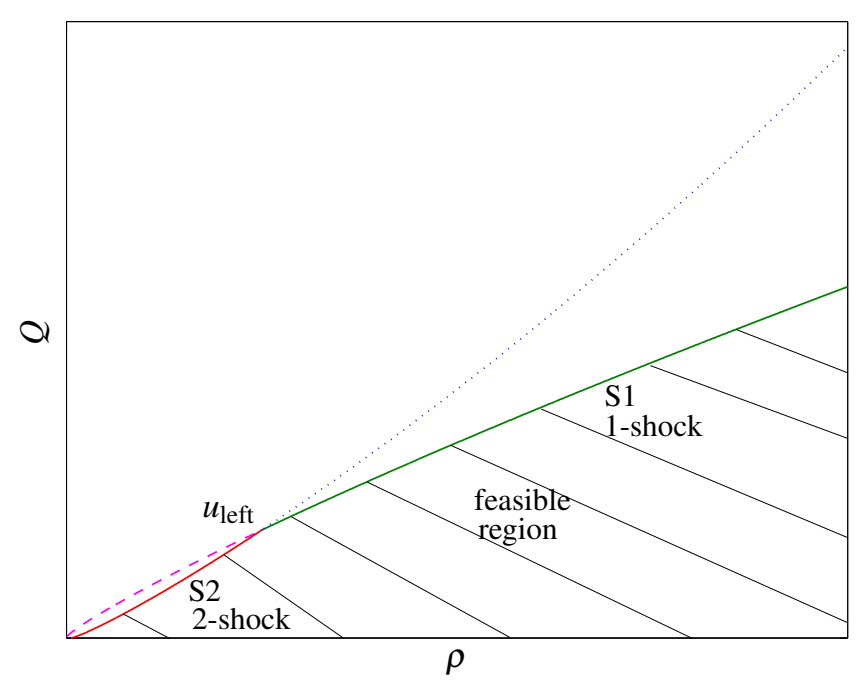

Fig. 1. Representation of the states $u_{\text {right }}$ that can be connected to $u_{\text {left }}$ by an entropy-satisfying shock. The dotted and dashed curves represent entropy-violating points.

two families of smooth solutions, called 1-rarefaction waves and 2-rarefaction waves exist. Similar to the analysis of shock curves we shall derive the phase curves for both families of rarefaction waves.

If $u(x, t)$ is a solution of the system (10), then we can show that $u(a x, a t)$ is also a solution, where $a$ is a scalar, i.e., the solutions are scaling-invariant. Therefore, the solution depends on $(x, t)$ in the form $\xi=x / t$. A rarefaction wave solution to the system of equations (7) takes the form:

$$
u(x, t)= \begin{cases}u_{\text {left }} & \text { if } \quad x \leq \xi_{1} t \\ w(x / t) & \text { if } \quad \xi_{1} t<x<\xi_{2} t \\ u_{\text {right }} & \text { if } \quad x \geq \xi_{2} t\end{cases}
$$

with $w(\cdot)$ smooth and $w\left(\xi_{1}\right)=u_{\text {left }}$ and $w\left(\xi_{2}\right)=u_{\text {right }}$. We will now prove that starting at each point $u_{\text {left }}$ there are two curves consisting of points $u_{\text {right }}$ that can be connected to $u_{\text {left }}$ by a rarefaction wave, namely a subset of the integral curve $^{3}$ of $r_{p}\left(u_{\text {left }}\right)$. We can determine explicitly the function $w(x / t)$ using the fact that our model is genuinely nonlinear. After some steps (see also [9]) we get the following differential equation:

$$
w^{\prime}(\xi)=\frac{r_{p}(w(\xi))}{\nabla \lambda_{p}(w(\xi)) \cdot r_{p}(w(\xi)} \quad \text { for } \quad \xi_{1} \leq \xi \leq \xi_{2},
$$

with initial conditions

$$
w\left(\xi_{1}\right)=u_{\text {left }}, \xi_{1}=\lambda_{p}\left(u_{\text {left }}\right)<\xi_{2}=\lambda_{p}\left(u_{\text {right }}\right) .
$$

For a 1-rarefaction wave we have:

$$
\frac{d}{d \xi} \rho(\xi)=\frac{\rho^{2}(\xi)}{Q(\xi)} \cdot \frac{1}{c_{1}^{2}-c_{1}}, \quad \frac{d}{d \xi} Q(\xi)=\rho(\xi) \frac{1}{c_{1}-1},
$$

${ }^{3}$ The integral curve for $r_{p}(u)$ is a curve that has the property that the tangent to the curve at any point $u$ lies in the direction $r_{p}(u)$. with $Q\left(\xi_{1}\right)=Q_{\text {left }}, \rho\left(\xi_{1}\right)=\rho_{\text {left }}, \xi_{1}=\lambda_{1}\left(u_{\text {left }}\right)=c_{1} \frac{Q_{\text {left }}}{\rho_{\text {left }}}$, which is a system of two ordinary nonlinear differential equations, with the following solution:

$$
\rho(\xi)=\left(\frac{\rho_{\text {left }}^{c_{1}}}{c_{1} Q_{\text {left }}} \cdot \xi\right)^{\frac{1}{c_{1}-1}}, Q(\xi)=\frac{\xi}{c_{1}}\left(\frac{\rho_{\text {left }}^{c_{1}}}{c_{1} Q_{\text {left }}} \cdot \xi\right)^{\frac{1}{c_{1}-1}} .
$$

If we want to obtain an explicit expression for the integral curves in the phase plane, we eliminate $\xi$, obtaining:

$$
Q(\rho)=Q_{\text {left }}\left(\frac{\rho}{\rho_{\text {left }}}\right)^{c_{1}}
$$

We can construct the 2-rarefaction wave in exactly the same manner obtaining:

$$
Q(\rho)=Q_{\text {left }}\left(\frac{\rho}{\rho_{\text {left }}}\right)^{c_{2}} .
$$

Now two states $u_{\text {left }}$ and $u_{\text {right }}$ can be connected by a rarefaction wave provided that they lie on the same integral curve and $\lambda_{p}\left(u_{\text {left }}\right)<\lambda_{p}\left(u_{\text {right }}\right)$, which for 1-rarefaction results in

$$
c_{1} \cdot \frac{Q_{\text {left }}}{\rho_{\text {left }}}<c_{1} \cdot \frac{Q_{\text {right }}}{\rho_{\text {right }}}, \quad c_{1} \in(0,1),
$$

with $Q_{\text {right }}=Q_{\text {left }}\left(\frac{\rho_{\text {right }}}{\rho_{\text {left }}}\right)^{c_{1}}$ and thus $\rho_{\text {left }}^{c_{1}-1}<\rho_{\text {right }}^{c_{1}-1}$ or $\rho_{\text {right }}<\rho_{\text {left }}$ since $c_{1} \in(0,1)$.

Therefore, the 1-rarefaction curve is given by

$$
\text { R1 : } \quad Q_{\text {right }}=Q_{\text {left }}\left(\frac{\rho_{\text {right }}}{\rho_{\text {left }}}\right)^{c_{1}}, \quad \rho_{\text {right }}<\rho_{\text {left }} .
$$

Similarly, the 2-rarefaction curve is given by

$$
\text { R2 : } \quad Q_{\text {right }}=Q_{\text {left }}\left(\frac{\rho_{\text {right }}}{\rho_{\text {left }}}\right)^{c_{2}}, \quad \rho_{\text {right }}>\rho_{\text {left }} .
$$

Figure 2 shows the states $u_{\text {right }}$ that can be connected to $u_{\text {left }}$ by a 1-rarefaction wave, namely the states lying on the curve R1 passing through $u_{\text {left }}$. Furthermore, the states $u_{\text {right }}$ lying on the curve R2 passing through $u_{\text {left }}$ can be connected to $u_{\text {left }}$ by a 2-rarefaction wave. Again we see that we do not connect negative states to $u_{\text {left }}$ and we will use this result in the next section when we discuss the Riemann problem.

\section{GENERAL SOLUTION OF THE RIEMANN PROBLEM}

In this section we discuss the Riemann problem associated to the Helbing's model and based on the results of the two previous sections we will show that the solutions of the Riemann problem with density and flow non-negative in the initial conditions on either side of the discontinuity cannot give rise to negative flows or density in the general solution. If we combine Figures 1 and 2 we obtain a plot that shows us all points $u_{\text {right }}$ that can be connected to a given point $u_{\text {left }}$ by an entropy-satisfying wave, either a shock wave or a rarefaction wave. Therefore, when initial data $u_{\text {left }}$ and $u_{\text {right }}$ both lay on these curves, then this discontinuity simply propagates with speed $s=\frac{Q_{\text {right }}-Q_{\text {left }}}{\rho_{\text {right }}-\rho_{\text {left }}}$ along the road. 


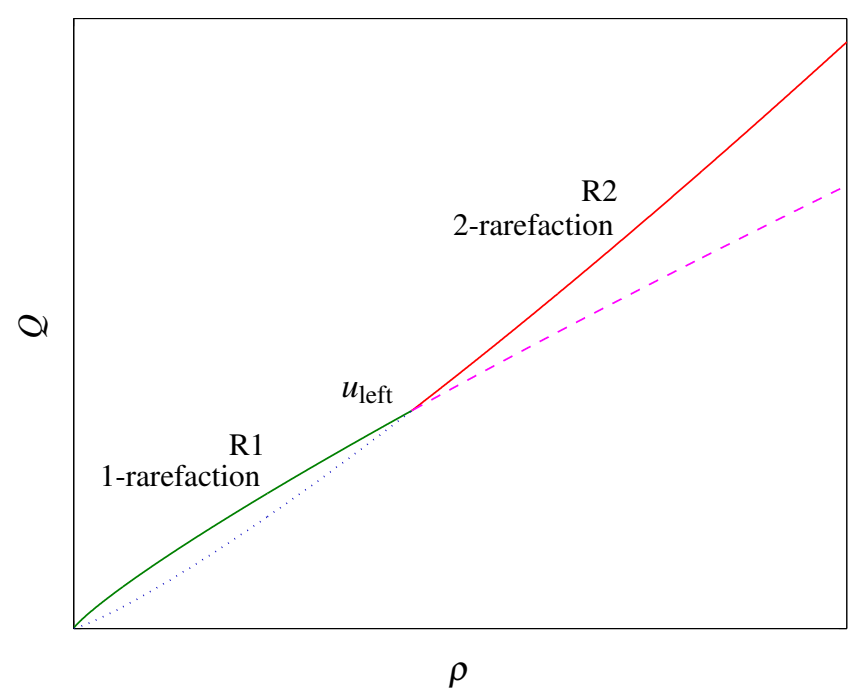

Fig. 2. Representation of the states $u_{\text {right }}$ that can be connected to $u_{\text {left }}$ by a rarefaction wave. The dotted and dashed curves represent points that do not satisfy the rarefaction condition (13).

But what happens if $u_{\text {right }}$ does not reside on one of those curves passing through $u_{\text {left }}$ ? To solve this question, we can attempt to find a way to split this jump as a sum of two jumps, across each of which the Rankine-Hugoniot condition holds, i.e., we must find an intermediate state $u_{\text {middle }}$ such that $u_{\text {left }}$ and $u_{\text {middle }}$ are connected by a discontinuity satisfying the Rankine-Hugoniot condition and so are $u_{\text {middle }}$ and $u_{\text {right }}$, which intuitively means to superimpose the appropriate plots and look for the intersections. When we want to determine analytically the intermediate state $u_{\text {middle, }}$ we must first determine whether each wave is a shock or a rarefaction, and then use the expressions relating $\rho$ and $Q$ determined in Sections III and IV along each curve to solve for the intersection. When we solve the equation given by the intersection, we can get more than one solution for $u_{\text {middle }}$ but only one gives a physically valid solution to the Riemann problem since the jump from $u_{\text {left }}$ to $u_{\text {middle }}$ must travel more slowly than the jump from $u_{\text {middle }}$ to $u_{\text {right }}$ (due to the condition $\lambda_{1}<\lambda_{2}$ ). Using the same parametrization as in Section III: $\rho_{\text {left }}=\rho_{\text {middle }}\left(1+\xi_{1}\right)$ and $\rho_{\text {right }}=\rho_{\text {middle }}\left(1+\xi_{2}\right)$, and replacing in (12) we get that the speeds of shock from $u_{\text {left }}$ to $u_{\text {middle }}$ and from $u_{\text {middle }}$ to $u_{\text {right }}$ are given by:

$$
\begin{gathered}
s_{\text {left }, \text { middle }}=\frac{Q_{\text {middle }}}{\rho_{\text {middle }}} \frac{\frac{1+c}{1+\xi_{1}} \pm \sqrt{\frac{c^{2}+c}{1+\xi_{1}}}}{1-\frac{\xi_{1}(1+c)}{1+\xi_{1}}}, \\
s_{\text {middle,right }}=\frac{Q_{\text {middle }}}{\rho_{\text {middle }}} \frac{\frac{1+c}{1+\xi_{2}} \pm \sqrt{\frac{c^{2}+c}{1+\xi_{2}}}}{1-\frac{\xi_{2}(1+c)}{1+\xi_{2}}} .
\end{gathered}
$$

Now depending on what values we choose for $u_{\text {left }}$ and $u_{\text {right }}$ we can determine the sign in the previous formulas such that $s_{\text {left,middle }}<s_{\text {middle,right }}$ and thus we know what waves (1-wave or 2-wave) give the intersection. We can distinguish the following cases:

Case 1: Both curves are shocks.

Graphically this means to draw the Hugoniot locus for each of the states $u_{\text {left }}$ and $u_{\text {right }}$ and to look for the intersection. To obtain the correct value for $u_{\text {middle }}=\left[\begin{array}{ll}\rho_{\text {middle }} & Q_{\text {middle }}\end{array}\right]^{T}$ we have to impose $s_{\text {left,middle }}<s_{\text {middle,right. Let }}$ us consider an example; e.g., assume that $u_{\text {middle }}$ is connected to $u_{\text {left }}$ by a 1 -shock and to $u_{\text {right }}$ by a 2 -shock:

$$
\begin{aligned}
& Q_{\text {middle }}=Q_{\text {left }} \frac{1-\left(\rho_{\text {middle }}-\rho_{\text {left }}\right) \sqrt{\frac{c^{2}+c}{\rho_{\text {middle }} \rho_{\text {left }}}}}{1-\frac{\rho_{\text {middle }}-\rho_{\text {left }}(1+c)}{\rho_{\text {middle }}}}, \\
& Q_{\text {middle }}=Q_{\text {right }} \frac{1+\left(\rho_{\text {middle }}-\rho_{\text {right }}\right) \sqrt{\frac{c^{2}+c}{\rho_{\text {middle }} \rho_{\text {right }}}}}{1-\frac{\rho_{\text {middle }}-\rho_{\text {right }}}{\rho_{\text {middle }}}(1+c)} .
\end{aligned}
$$

Equating the two right-hand sides gives a single equation for $\rho_{\text {middle }}$. After we obtain $\rho_{\text {middle }}$, we replace it in (15) or (16) to obtain $Q_{\text {middle }}$.

When $u_{\text {middle }}$ is connected to $u_{\text {left }}$ by a 2 -shock and to $u_{\text {right }}$ by a 1 -shock we proceed similarly.

Case 2: Both curves are rarefactions.

If we assume that the intermediate state is connected to $u_{\text {left }}$ by a 1-rarefaction and to $u_{\text {right }}$ by a 2-rarefaction, then $u_{\text {middle }}$ must satisfy

$$
\begin{aligned}
& Q_{\text {middle }}=Q_{\text {left }}\left(\frac{\rho_{\text {middle }}}{\rho_{\text {left }}}\right)^{c_{1}}, \\
& Q_{\text {middle }}=Q_{\text {right }}\left(\frac{\rho_{\text {middle }}}{\rho_{\text {right }}}\right)^{c_{2}} .
\end{aligned}
$$

Equating again we get an equation in $\rho_{\text {middle }}$ with solution

$$
\rho_{\text {middle }}=\left(\frac{Q_{\text {left }}}{Q_{\text {right }}} \frac{\rho_{\text {right }}^{c_{2}}}{\rho_{\text {left }}^{c_{1}}}\right)^{\frac{1}{c_{2}-c_{1}}}
$$

and then we obtain $Q_{\text {middle }}$ from the previous equations.

Case 3: The solution consist of one shock and one rarefaction wave.

Again if we consider the case when the intermediate state $u_{\text {middle }}$ is connected to $u_{\text {left }}$ by a 1 rarefaction and to $u_{\text {right }}$ by a 2 -shock, then we must solve for $\rho_{\text {middle }}$ and $Q_{\text {middle }}$ from the following system of equations:

$$
\begin{aligned}
& Q_{\text {middle }}=Q_{\text {left }} \frac{1+\left(\rho_{\text {middle }}-\rho_{\text {left }}\right) \sqrt{\frac{c^{2}+c}{\rho_{\text {middle }} \rho_{\text {left }}}}}{1-\frac{\rho_{\text {middle }}-\rho_{\text {left }}}{\rho_{\text {middle }}}(1+c)}, \\
& Q_{\text {middle }}=Q_{\text {right }}\left(\frac{\rho_{\text {middle }}}{\rho_{\text {right }}}\right)^{c_{1}} .
\end{aligned}
$$

Figure 3 shows a plot for the Riemann problem with initial conditions $u_{\mathrm{left}}=\left[\begin{array}{ll}140 & 400\end{array}\right]^{T}$ and $u_{\text {right }}=\left[\begin{array}{ll}5 & 50\end{array}\right]^{T}$, 


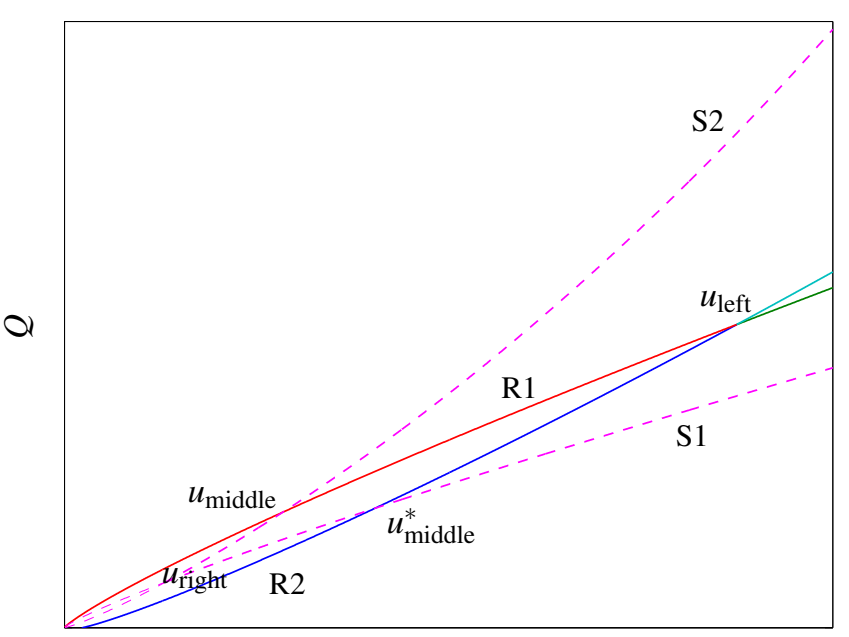

$\rho$

Fig. 3. Construction of the solution for the Riemann problem. We obtain two intermediate states $u_{\text {middle }}$ and $u_{\text {middle }}^{*}$, but only $u_{\text {middle }}^{*}$ is a physically valid solution.

which corresponds, e.g., to a scenario such as the situation of traffic in front of a semaphore when it was red and then becomes green. Lines represent the states that can be connected to $u_{\text {left }}$ and dotted curves represent the states that can be connected to $u_{\text {right }}$. The intersection gives two points but only one is a physically valid solution because we should have $s_{\text {left,middle }}<s_{\text {middle,right }}$ (due to $\lambda_{1}<\lambda_{2}$ ). The intermediate state $u_{\text {middle }}$ is obtained by the intersection of $\mathrm{R} 1$ with $\mathrm{S} 2$, and $u_{\text {middle }}^{*}$ by the intersection of $\mathrm{S} 2$ with $\mathrm{R} 1$. In this case $u_{\text {middle }}^{*}$ is the physically valid solution.

\section{CONCLUSIONS AND FUTURE RESEARCH}

In this paper we have discussed some properties of Helbing's gas-kinetic traffic flow model. More specifically, we have derived formulas for shocks and rarefaction waves and we have characterized the states that satisfy the Lax entropy condition. Understanding structural properties of the model allow us to choose the appropriate numerical scheme for simulations and further for applications such as model-based traffic control. Finally, we have considered the Riemann problem associated with the Helbing's model when the traffic conditions are in equilibrium, proving that the solution of this problem with density and flow non-negative in the initial condition on either side of the discontinuity cannot give rise to negative flow or density later on.

Topics for further research include: investigation of appropriate efficient numerical schemes to simulate Helbing's model and development of model-based traffic control techniques for traffic using Helbing's model.

\section{ACKNOWLEDGMENTS}

Research supported by the NWO-CONNEKT project "Advanced multi-agent control and information for integrated multi-class traffic networks", the STW projects
"Model predictive control for hybrid systems" and "Multiagent control of large-scale hybrid systems", and the TU Delft Spearhead Program "Mobility of People and Transportation of Goods".

\section{REFERENCES}

[1] M. Lighthill and G. Whitham, "On kinematic waves: II. A theory of traffic flow on long crowded roads," Proceedings of the Royal Society of London, vol. 299A, pp. 317-345, May 1955.

[2] H. Payne, "Models of freeway traffic and control," in Mathematical Models of Public Systems, ser. Simulation Council Proceedings Series, G. Bekey, Ed. La Jolla, California, 1971, vol. 1, no. 1, pp. 51-61.

[3] M. Papageorgiou, J. Blosseville, and H. Haj-Salem, "Modelling and real-time control of traffic flow on the southern part of Boulevard Périphérique in Paris: Part I: Modelling," Transportation Research Part A, vol. 24, no. 5, pp. 345-359, Sept. 1990.

[4] D. Helbing, "Derivation, properties, and simulation of a gas-kineticbased, nonlocal traffic model," Physical Review E, vol. 59, no. 1, pp. 239-253, 1999.

[5] S. Hoogendoorn and P. Bovy, "Generic gas-kinetic traffic systems modeling with applications to vehicular traffic flow," Transportation Research Part B, vol. 35, no. 4, pp. 317-336, May 2001.

[6] S. Paveri-Fontana, "On Boltzmann-like treatments for traffic flow. A critical review of the basic model and an alternative proposal for dilute traffic analysis," Transportation Research, vol. 9, pp. 225-235, 1975.

[7] I. Prigogine and R. Hermann, Kinetic Theory Of Vehicular Traffic New York: Elsevier, 1971.

[8] R. LeVeque, Numerical Methods for Conservation Laws, ser. Lectures in Mathematics. Birkhauser, 1992.

[9] I. Necoara, B. De Schutter, and J. Hellendoorn, "Structural properties of Helbing's traffic flow model," in Proceedings of the 83rd Annual Meeting of the Transportation Research Board, Washington, D.C., Jan. 2004, paper 04-2263.

[10] P. Lax, Hyperbolic Systems of Conservation Laws and the Mathematical Theory of Shock Waves. Philadelphia, PA: Society for Industrial and Applied Mathematics, 1973.

[11] A. May, Traffic Flow Fundamentals. Englewood Cliffs, New Jersey: Prentice-Hall, 1990

[12] C. Daganzo, "Requiem for second-order fluid approximations of traffic flow," Transportation Research Part B, vol. 29, no. 4, pp. 277-286, Aug. 1995. 